\title{
Complete Genome Sequence Data of Four Burkholderia glumae Strains Isolated from Rice Fields in the United States
}

\author{
Hyun-Hee Lee, ${ }^{1}$ Tiago Lelis, ${ }^{2}$ John Ontoy, ${ }^{2}$ Jobelle Bruno, ${ }^{2}$ Jong Hyun Ham, ${ }^{2, \dagger}$ and \\ Young-Su Seo ${ }^{1, \dagger}$ \\ ${ }^{1}$ Department of Integrated Biological Science, Pusan National University, Busan 46247, Korea \\ ${ }^{2}$ Department of Plant Pathology and Crop Physiology, Louisiana State University Agricultural \\ Center, Baton Rouge, LA 70803, U.S.A.
}

\begin{abstract}
Bacterial panicle blight caused by Burkholderia glumae is a major disease in rice production worldwide. Currently, only a few whole-genome sequences of $B$. glumae strains isolated in the United States are available. Here, we report the complete genome sequence of four $B$. glumae strains, including three virulent strains (336gr-1, 411gr-6, and 95785641-c) and the nonpathogenic strain B. glumae 257sh-1, which were isolated from rice fields in Louisiana (336gr-1, 957856-41-c, and 257sh-1) and Arkansas (411gr-6). The whole-genome sequence data of $B$. glumae strains will contribute to investigations of the molecular mechanism underlying bacterial pathogenicity and virulence to rice plants.
\end{abstract}

\section{Genome Announcement}

Burkholderia glumae was first reported in Japan as a causal agent for seedling blight and grain rotting (Goto and Ohata 1956). In the United States, bacterial panicle bight caused by $B$. glumae is the primary bacterial disease of rice (Ham et al. 2011). Bacterial panicle blight is also a frequent problem in several rice-producing areas worldwide (Cui et al. 2016). The pathogen infection leads to the sterility of the spikelet and discoloration of the emerging grains, affecting the quantity and quality of rice grains (Nandakumar et al. 2009). The virulence of $B$. glumae to rice plants requires numerous virulence factors such as toxoflavin, lipase, type III secretion effectors, catalase, and extracellular protease (Chun et al. 2009; Devescovi et al. 2007; Kim et al. 2004; Lelis et al. 2019). B. glumae strains are phenotypically diverse, infecting many plant species ranging from monocot to dicot (Jeong et al. 2003). Strains isolated from symptomatic rice plants showed variation in virulence, toxin, and pigment production (Karki et al. 2012). Some pigment-producing strains of B. glumae showed an intense antifungal activity against Colletotrichum orbiculare (Karki et al. 2012). Despite the economic impact caused by $B$. glumae on rice production worldwide, only a few genomes of $B$. glumae were fully sequenced and assembled.

${ }^{\dagger}$ Corresponding authors: J. H. Ham; jham @ agcenter.Isu.edu; and Y.-S. Seo; yseo2011 @ pusan.ac.kr H.-H. Lee and T. Lelis contributed equally to this work.

Current address for T. Lelis: Tropical Research and Education Center, University of Florida, Homestead, FL 33031, U.S.A.

The author(s) declare no conflict of interest.

Accepted for publication 3 August 2021.

\section{Funding}

This research was supported by the Basic Science Research Program through the National Research Foundation of Korea funded by the Ministry of Education (grant 2019R1A2C2006779), the Louisiana Rice Research Board (GR-00006499), and the United States Department of Agriculture-National Institute of Food and Agriculture (Hatch project 1015305). T. Lelis was supported by the Conselho Nacional de Desenvolvimento Cientifico e Tecnologico Science Without Border program of the Brazilian Government.

\section{Keywords}

bacterial panicle blight, Burkholderia glumae, rice 


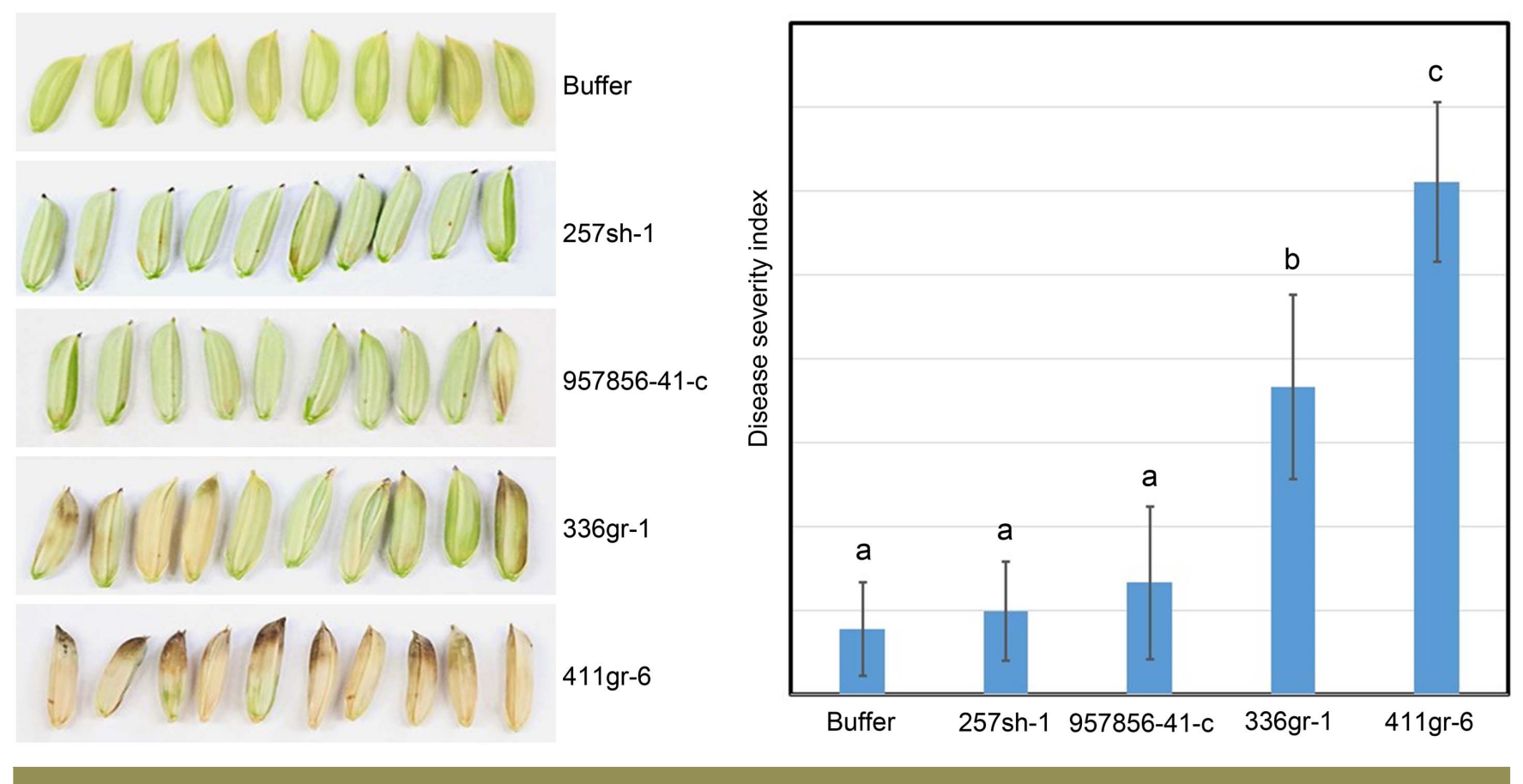

Fig. 1. Virulence phenotypes of Burkholderia glumae 257sh-1, B. glumae 957856-41-c, B. glumae 336gr-1, and B. glumae 411gr-6. The disease severity index used in this study was based on a 0-to-9 scale, where $0=$ no symptoms and $9=$ more than $90 \%$ of symptomatic area in each panicle. Each data point represents the average value of three replications (one replication $=$ three plants in each pot). Significant differences among the data points were determined at $P<0.05$ using Tukey's honestly significant difference test. For inoculation, immature rice panicles were sprayed with the bacterial suspension of each strain at the concentration of approximately $5 \times 10^{7} \mathrm{CFU} / \mathrm{ml}$ (optical density at $600 \mathrm{~nm}=0.1$ ). After inoculation, plants were maintained in the greenhouse room until the completion of data collection. Photos were taken at 11 days after bacterial inoculation.

Here, we report the whole-genome sequence data of four B. glumae strains showing different phenotypic traits in virulence and metabolism: 257sh-1, 336gr-1, 411gr-6, and 957856-41-c. B. glumae 257sh-1 is a natural nonpathogenic and pigment-producing strain from Louisiana (Karki et al. 2012). B. glumae 336gr-1 is a virulent and non-pigment-producing strain from Louisiana (Karki et al. 2012). B. glumae 957856-41-c was previously reported as a virulent and non-pigment-producing strain from Louisiana (Karki et al. 2012). However, this strain produced a much lesser amount of the major virulence factor, toxoflavin, compared with other virulent strains (Karki 2010). We reevaluated the virulence of $B$. glumae 957856-41-c along with other strains sequenced in this study, in which this strain was almost nonpathogenic showing no significant difference from the nonpathogenic strain, $B$. glumae 257sh-1 (Fig. 1). B. glumae $411 \mathrm{gr}-6$ is a highly virulent and pigment-producing strain from Arkansas (Karki et al. 2012). The virulence phenotypes of the four strains sequenced in this study are presented in Figure 1.

Genomic DNA was extracted using the GenElute Bacterial Genomic DNA Extraction kit (Sigma-Aldrich) following manufacturer protocols. NanoDrop ND-100 Spectrophotometer (NanoDrop Technologies, Inc., Wilmington, DE, U.S.A.) was used to assess the quality and quantity of the DNA sample. Whole-genome sequencing library was prepared with a SMRTbell template prep kit (Pacific Biosciences, Menlo Park, CA, U.S.A.). Single-molecule real-time (SMRT) sequencing was conducted on a PacBio RS II platform (Pacific Biosciences) by Macrogen (Seoul, South Korea) for strains 336gr-1, 411gr-6, and 957856-41-c, and ChunLab (Seoul, South Korea) for strain 257sh-1. De novo assembly with the hierarchical genome assembly process (HGAP) version 3 was implemented in the PacBio SMRT Analysis algorithm version 2.3.0 package (Chin et al. 2013). The HGAP data processing is consisted of PreAssembler for filtering, Celera assembler for assembly, and BLASR for mapping (Chin et al. 2013). The initial assembly was followed by two rounds of polishing by Quiver for further quality improvements of genome sequences (Chin et al. 2013). Gene prediction and annotation were initially performed by Prokka version 1.12 (Seemann 2014). The genome sequence was reannotated with the NCBI prokaryotic genome annotation pipeline (Tatusova 
Table 1. Genome features and statistics of the Burkholderia glumae genomes

\begin{tabular}{|c|c|c|c|c|}
\hline \multirow[b]{2}{*}{ Features } & \multicolumn{4}{|c|}{ B. glumae strains } \\
\hline & $336 g r-1$ & $411 \mathrm{gr}-6$ & 957856-41-c & $257 \mathrm{sh}-1$ \\
\hline Chromosome & 2 & 2 & 2 & 2 \\
\hline Plasmid & 3 & 2 & 2 & 2 \\
\hline NCBI accession & СР023203-7 & СР021157-60 & СР021074-7 & СР035900-3 \\
\hline $\mathrm{N}_{50}$ & 3.60 & 3.60 & 3.76 & 3.54 \\
\hline Coverage & $110 X$ & $143 X$ & $128 X$ & $168 X$ \\
\hline Size $(\mathrm{Mb})$ & 6.88 & 6.75 & 6.95 & 6.69 \\
\hline GC (\%) & 68.12 & 68.23 & 68.10 & 68.19 \\
\hline Protein & 5896 & 5998 & 6004 & 5606 \\
\hline Ribosomal RNA & 15 & 15 & 15 & 15 \\
\hline Transfer RNA & 77 & 77 & 78 & 66 \\
\hline
\end{tabular}

Table 2. Predicted effector proteins secreted by type III (T3) secretion system in the Burkholderia glumae genomes

$\begin{array}{ll}\text { Strains, locus ID } & \begin{array}{c}\text { Conserved chaperone binding domain (CCBD) detection } \\ \text { by EffectiveCCBD }\end{array} \\ \text { 336gr-1 } & \\ \text { CG017_RS05450 } & \text { CCBD (ITPAVSFVVN) found downstream of expected region } \\ \text { CG017_RS28175 } & \text { CCBD (LGRDVLDVVN) found downstream of expected region } \\ \text { s11gr-6 } & \text { CCBD (ITPAVSFVVN) found downstream of expected region } \\ \text { B7760_RS15050 } & \text { CCBD (LGRDVLDVN) found downstream of expected region } \\ \text { B7760_RS27555 } & \text { CCBD (LRESIQRVWN) found downstream of expected region } \\ \text { 957856-41-C } & \text { CCBD (ITPAVSFVVN) found downstream of expected region } \\ \text { B7759_RS06100 } & \text { CCBD (LGRDVLDVN) found downstream of expected region } \\ \text { B7759_RS16550 } & \text { CCBD (IYLPIYFVSN) found within expected region } \\ \text { B7759_RS20495 } & \text { CCBD (ITPAVSFVVN) found downstream of expected region } \\ \text { B7759_RS24610 } & \text { CCBD (LGRDVLDVVN) found downstream of expected region } \\ \text { 257sh-1 } & \\ \text { EXE55_RS10890 } & 1 \\ \text { EXE55_RS22555 } & \end{array}$

et al. 2016) based on the best-placed reference protein set and, finally, deposited in the NCBI database.

The detailed genome features and statistics of four $B$. glumae strains are summarized in Table 1. All B. glumae strains (336gr-1, 411gr-6, 957856-41-C, and 257sh-1) harbored two circular chromosomes (size ranging from 3,761,157 to 3,537,953 bp), and two plasmids (size ranging from 193,251 to 135,963 bp), except B. glumae 336gr-1, which carried additional plasmid (size 111,135 bp). In addition, the four $B$. glumae genomes were analyzed for putative type III secretion systems (T3SS) and effector proteins of T3SS. The EffectiveDB server was used for identification of the potential T3SS with EffectiveS346, identification of effector proteins with EffectiveT3 for prediction of secretion signals, and conserved chaperone binding domain detection with EffectiveCCBD (Eichinger et al. 2016). All genomes of four B. glumae strains encoded genes for T3SS and effector proteins. In particular, Table 2 shows the potential effector proteins which were predicted by both EffectiveT3 and EffectiveCCBD tools (Eichinger et al. 2016). The whole-genome sequence reported here will contribute not only to our understanding of $B$. glumae genomic variation but also to development of strategies to prevent and control bacterial panicle bight caused by $B$. glumae.

\section{Acknowledgments}

We thank $\mathrm{H}$. Son for his advice on genome analyses and I. Barphagha for technical support. 


\section{Literature Cited}

Chin, C. S., Alexander, D. H., Marks, P., Klammer, A. A., Drake, J., Heiner, C., Clum, A., Copeland, A., Huddleston, J., Eichler, E. E., Turner, S. W., and Korlach, J. 2013. Nonhybrid, finished microbial genome assemblies from long-read SMRT sequencing data. Nat. Methods 10:563-569.

Chun, H., Choi, O., Goo, E., Kim, N., Kim, H., Kang, Y., Kim, J., Moon, J. S., and Hwang, I. 2009. The quorum sensing-dependent gene katG of Burkholderia glumae is important for protection from visible light. J. Bacteriol. 191:4152-4157.

Cui, Z.-q., Zhu, B., Xie, G.-I., Li, B., and Huang, S.-w. 2016. Research status and prospect of Burkholderia glumae, the pathogen causing bacterial panicle blight. Rice Sci. 23:111-118.

Devescovi, G., Bigirimana, J., Degrassi, G., Cabrio, L., LiPuma, J. J., Kim, J., Hwang, I., and Venturi, V. 2007. Involvement of a quorum-sensing-regulated lipase secreted by a clinical isolate of Burkholderia glumae in severe disease symptoms in rice. Appl. Environ. Microbiol. 73:4950-4958.

Eichinger, V., Nussbaumer, T., Platzer, A., Jehl, M. A., Arnold, R., and Rattei, T. 2016. EffectiveDB-Updates and novel features for a better annotation of bacterial secreted proteins and type III, IV, VI secretion systems. Nucleic Acids Res. 44:D669-D674.

Goto, K., and Ohata, K. 1956. New bacterial diseases of rice (brown stripe and grain rot). Ann. Phytopathol. Soc. Jpn. 21:46-47.

Ham, J. H., Melanson, R. A., and Rush, M. C. 2011. Burkholderia glumae: Next major pathogen of rice? Mol. Plant Pathol. 12:329-339.

Jeong, Y., Kim, J., Kim, S., Kang, Y., Nagamatsu, T., and Hwang, I. 2003. Toxoflavin produced by Burkholderia glumae causing rice grain rot is responsible for inducing bacterial wilt in many field crops. Plant Dis. 87: 890-895.

Karki, H. S. 2010. Physiological, biochemical and molecular characteristics associated with virulence of Burkholderia glumae: The major causative agent of bacterial panicle blight of rice. Master's thesis, Louisiana State University.

Karki, H. S., Shrestha, B. K., Han, J. W., Groth, D. E., Barphagha, I. K., Rush, M. C., Melanson, R. A., Kim, B. S., and Ham, J. H. 2012. Diversities in virulence, antifungal activity, pigmentation and DNA fingerprint among strains of Burkholderia glumae. PLoS One 7:e45376.

Kim, J., Kim, J. G., Kang, Y., Jang, J. Y., Jog, G. J., Lim, J. Y., Kim, S., Suga, H., Nagamatsu, T., and Hwang, I. 2004. Quorum sensing and the LysR-type transcriptional activator ToxR regulate toxoflavin biosynthesis and transport in Burkholderia glumae. Mol. Microbiol. 54:921-934.

Lelis, T., Peng, J., Barphagha, I., Chen, R., and Ham, J. H. 2019. The virulence function and regulation of the metalloprotease gene prtA in the plantpathogenic bacterium Burkholderia glumae. Mol. Plant-Microbe Interact. 32: 841-852.

Nandakumar, R., Shahjahan, A. K. M., Yuan, X. L., Dickstein, E. R., Groth, D. E., Clark, C. A., Cartwright, R. D., and Rush, M. C. 2009. Burkholderia glumae and B. gladioli cause bacterial panicle blight in rice in the Southern United States. Plant Dis. 93:896-905.

Seemann, T. 2014. Prokka: Rapid prokaryotic genome annotation. Bioinformatics 30:2068-2069.

Tatusova, T., DiCuccio, M., Badretdin, A., Chetvernin, V., Nawrocki, E. P., Zaslavsky, L., Lomsadze, A., Pruitt, K. D., Borodovsky, M., and Ostell, J. 2016. NCBI prokaryotic genome annotation pipeline. Nucleic Acids Res. 44:6614-6624. 